\title{
Recurrent hypoglycemia inhibits the counterregulatory response by suppressing adrenal activity
}

\author{
Yunbing Ma, Qian Wang, Debria Joe, Manqi Wang, and Matthew D. Whim \\ Department of Cell Biology and Anatomy, LSU Health Sciences Center, New Orleans, Louisiana, USA.
}

\begin{abstract}
Hypoglycemia activates the counterregulatory response (CRR), a neural-endocrine reflex that restores euglycemia. Although effective if occasionally activated, repeated induction of the CRR leads to a decline in responsiveness and prolonged exposure to hypoglycemia. The mechanism underlying this impairment is not known. We found that the reduction in epinephrine release that characterizes a suppressed CRR involves a long-lasting form of sympatho-adrenal synaptic plasticity. Using optogenetically evoked catecholamine release, we show that recurrent hypoglycemia reduced the secretory capacity of mouse adrenal chromaffin cells. Single activation of the CRR increased the adrenal levels of tyrosine hydroxylase (TH), the rate-limiting enzyme for catecholamine synthesis, but this was prevented by repeated activation. In contrast, the level of neuropeptide Y (NPY), an adrenal cotransmitter, remained elevated after recurrent hypoglycemia. Inhibition of NPY or Y1 signaling, either transgenically or pharmacologically, prevented the attenuation of both TH expression and epinephrine release. These results indicate that impairment of the CRR involves suppressed activity at the adrenal level. Interfering with the peripheral NPY-dependent negative feedback loop may provide a way to avoid the pathophysiological consequences of recurrent hypoglycemia which are common in the diabetic state.
\end{abstract}

\section{Introduction}

In response to hypoglycemia, the counterregulatory response (CRR) restores euglycemia (1). The hormonal components of the CRR depend on the degree of hypoglycemia. Changes in insulin and glucagon release are normally sufficient to restore euglycemia, but in type 1 and type 2 diabetes, pancreatic islet function is impaired, and the CRR becomes critically dependent on epinephrine release. However, during repeated episodes of hypoglycemia, epinephrine secretion is progressively reduced, even in nondiabetic individuals (2). This activity-dependent failure of the CRR, together with an unawareness of hypoglycemia, is known as hypoglycemia-associated autonomic failure (HAAF) and is a major limitation to clinically effective glycemic control (1).

Epinephrine is secreted from neuroendocrine chromaffin cells in the adrenal medulla. The activity of these cells, which are part of the sympathetic nervous system, is controlled by spinal preganglionic neurons, which receive descending input from the rostral ventrolateral medulla. Once released into the circulation, epinephrine elevates hepatic glucose production through an increase in glycogenolysis and indirectly by increasing the supply of gluconeogenic substrates (3).

What causes HAAF is unclear, although several neurotransmitters and hormones have been implicated (4-7). Impairment of the CRR is associated with altered glucosensitivity of neurons in the ventromedial hypothalamus and in GABAergic tone $(8,9)$. The activity of hindbrain catecholamine neurons is also suppressed

Related Commentary: p. 3739

Conflict of interest: The authors have declared that no conflict of interest exists. Submitted: December 2, 2016; Accepted: June 20, 2018.

Reference information: J Clin Invest. 2018;128(9):3866-3871.

https://doi.org/10.1172/JCI91921. following repeated glucoprivation (10). Although these findings point to a central mechanism, this cannot fully explain the loss of the CRR. After recurrent hypoglycemia, adrenal nerve activity is not suppressed even though epinephrine release is reduced $(11,12)$, implying that a defect is located within the adrenal gland (13-15). Here, we show that the characteristic phenotype of an impaired CRR, namely a reduction in catecholamine release, involves peptidergic regulation of synaptic plasticity within the adrenal gland.

\section{Results and Discussion}

Recurrent activation of the CRR produces an autonomous, long-lasting change in adrenal function. To examine the effect of repeated activation of the CRR, mice received saline, a single insulin injection, or recurrent insulin injections (Figure 1A), and urine epinephrine levels were quantified. One hypoglycemic episode increased urine epinephrine, consistent with a functional CRR (control: $64 \pm 7 \mathrm{ng}$ / mg creatinine; insulin: $202 \pm 25 ; n=10$ ). Following recurrent hypoglycemia, the increase in epinephrine levels was reduced compared with levels following a single episode of hypoglycemia (129 $\pm 18 \mathrm{ng}$ / mg creatinine, $n=10$ ), and this increase was not statistically different from saline-injected controls, even though hypoglycemia was present after each insulin injection (Figure 1, B and C). When epinephrine levels were measured in plasma, the same trend was observed (Supplemental Figure 1, A and B; supplemental material available online with this article; https:/doi.org/10.1172/JCI91921DS1), and urine and plasma epinephrine levels were tightly correlated $(R=0.98$; Supplemental Figure 1C), even though the samples were collected at different time points after the onset of hypoglycemia (urine: $24 \mathrm{~h}$; plasma: 1 h). The suppression of epinephrine release after recurrent hypoglycemia was not due to a difference in insulin treatment between groups (16), because when the CRR was recurrently activated using DREADD (designer receptors exclusively activated by designer drugs) 
A

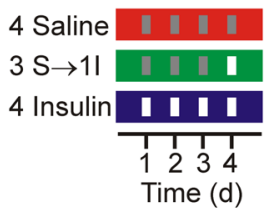

D

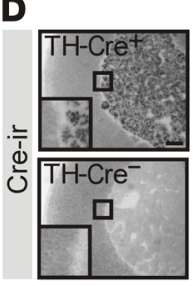

I

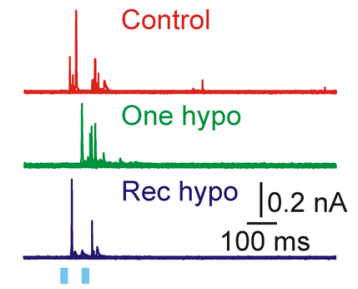

B

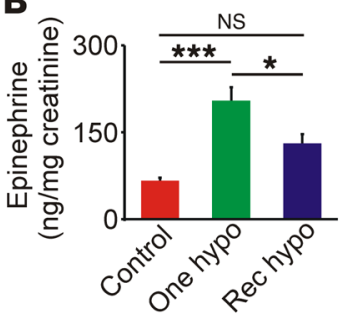

$\mathbf{F}$
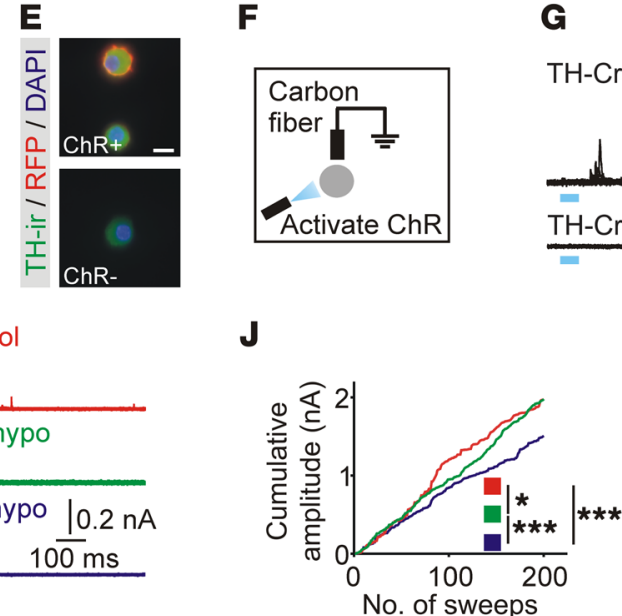

C
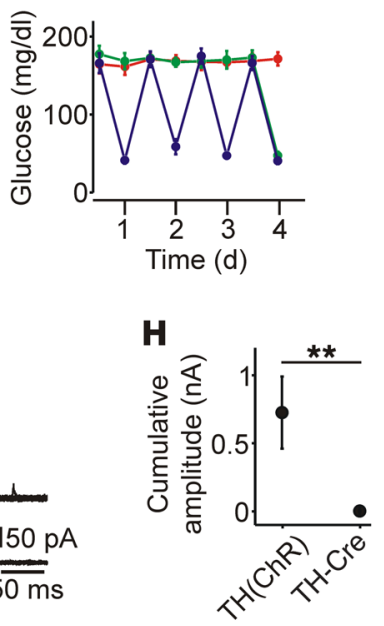

$\mathbf{K}$

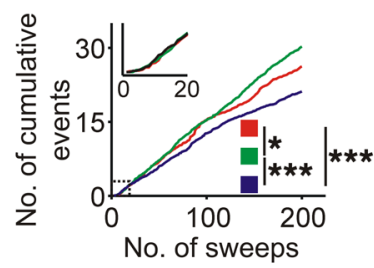

Figure 1. Recurrent episodes of hypoglycemia suppress catecholamine release from adrenal chromaffin cells. (A) Schematic of glycemic protocols. Red: recurrent saline (Control); green: recurrent saline plus insulin (One hypo); blue: recurrent insulin (Rec hypo); gray: saline injection; white: insulin injection. I, insulin; S, saline. (B) Hypoglycemia-induced epinephrine release in vivo was blunted after recurrent insulin injection $(n=10)$. (C) Insulin injection evoked a reproducible fall in blood glucose levels $(n=9-10)$. (D) Cre immunoreactivity (Cre-ir) was restricted to the adrenal medulla in TH-Cre+ mice. (E) In TH-ChR(tdTomato) mice, RFP fluorescence was present on the cell membrane of chromaffin cells. TH-ir, TH immunoreactivity. (F) Catecholamine release was evoked from chromaffin cells in vitro using ChR-mediated depolarization and detected using carbon fiber amperometry. (C) Example of optogenetically evoked amperometric events. (H) Catecholamine secretion was detected from TH-Cre+ chromaffin cells that also expressed ChR ( $n=12-13$ cells). (I) Examples of amperometric events from mice exposed to glycemic protocols. (J) Cumulative amplitude of amperometric events (control: 554 events; 1 hypoglycemia: 1,008 events; recurrent hypoglycemia: 597 events; $n=21-33$ cells per condition from 4 animals per treatment; plot was normalized to the number of recorded cells). (K) Cumulative number of amperometric events (from experiments in J). Scale bars: $100 \mu \mathrm{m}$ (D), $10 \mu \mathrm{m}$ (E). ${ }^{*} P<0.05,{ }^{* *} P<0.01$, and ${ }^{* * *} P<0.001$, by 1-way ANOVA (B), Wilcoxon rank-sum test (H), Kolmogorov-Smirnov test (J and $\mathbf{K})$.

technology (Supplemental Figure 2) or 2DG (Supplemental Figure 3), a subsequent insulin injection still evoked less release after antecedent CRR activation compared with that observed in mice in which the CRR had not previously been activated. Thus, this protocol (Figure 1A) produces an activity-dependent reduction in the CRR.

We next tested whether the reduced epinephrine release involved a functional change in chromaffin cells. To measure the latter, we used optogenetics to selectively depolarize chromaffin cells in vitro and monitored catecholamine release amperometrically. We found that channelrhodopsin $(\mathrm{ChR})$ was expressed in chromaffin cells using TH-Cre LSL-hChR2(tdTomato) mice. In adrenal cryosections, Cre immunoreactivity was restricted to the medulla (Figure 1D). Chromaffin cells from TH-ChR(tdTomato) mice in vitro were $\mathrm{TH}$ immunoreactive, and RFP fluorescence was localized at the plasma membrane (Figure 1E). We noted no relationship between the level of RFP fluorescence and the immunoreactivity of tyrosine hydroxylase (TH) or phenylethanolamine $N$-methyltransferase (PNMT) (Supplemental Figure 4), indicating that ChR expression was not restricted to a subset of chromaffin cells. Amperometric recordings in vitro showed that brief exposure to blue light evoked secretory events in cells from TH-ChR(tdTomato) mice but not from animals lacking ChR (Figure 1, F-H).
Next, secretion from chromaffin cells isolated from TH-ChR (tdTomato) mice exposed to the 3 glycemic protocols was measured in vitro (Figure 1I). Secretion was evoked by light flashes and quantified as the cumulative amplitude of amperometric events. Recurrent hypoglycemia led to a significant reduction in catecholamine release compared with that detected in cells from control mice or cells exposed to 1 hypoglycemic episode (Figure 1J). Consistent with a change in secretion, prior exposure to 1 episode of hypoglycemia led to an increase in the number of evoked events, while recurrent hypoglycemia led to a reduction in these events (Figure 1K). Thus the consequences of recurrent hypoglycemia can be detected in isolated chromaffin cells.

Insulin-induced hypoglycemia (IIH) can increase mRNA levels of $\mathrm{TH}$, the rate-limiting enzyme for catecholamine synthesis $(14,17)$, and could be a mechanism that couples hypoglycemia to a subsequent change in catecholamine secretory capacity. After 1 hypoglycemic episode, adrenal TH immunoreactivity was increased but was at basal levels after recurrent hypoglycemia (Figure 2, A-C). A similar differential effect of recurrent hypoglycemia on TH mRNA levels has also been observed $(13,14)$. We conclude that recurrent hypoglycemia either suppresses $\mathrm{TH}$ expression or prevents its repeated induction. 

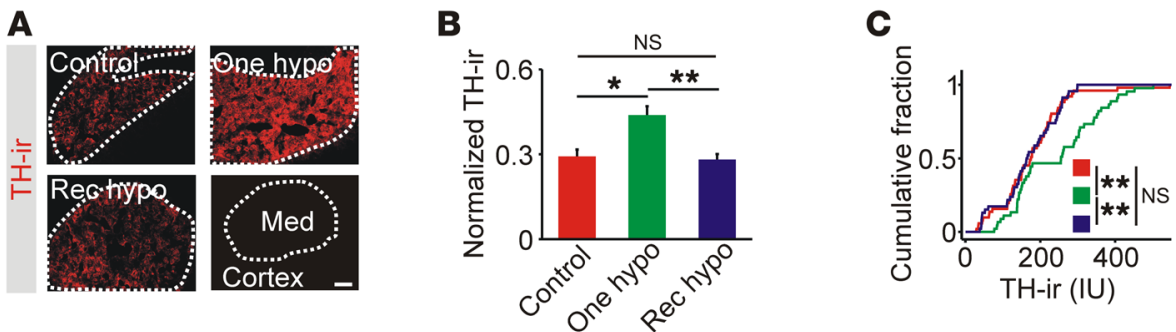

Figure 2. Adrenal levels of TH are elevated after single, but not recurrent, episodes of hypoglycemia. (A) TH immunoreactivity was restricted to the adrenal medulla (Med). Scale bar: $100 \mu \mathrm{m}$. (B) TH immunoreactivity was increased after 1 episode of hypoglycemia $(n=5)$. (C). Cumulative intensity distribution of TH immunoreactivity in all cryosections ( $n=8-14$ per experiment). ${ }^{*} P<0.05$ and ${ }^{* *} P<0.01$, by 1 -way ANOVA (B) and Kolmogorov-Smirnov test (C).

Neuropeptide $Y$ release mediates adrenal impairment of the CRR. Neuropeptide Y (NPY) is coreleased with the catecholamines from chromaffin cells, and $\mathrm{Y}$ receptors are expressed in the adrenal medulla $(18,19)$. We have shown that NPY acting via Y1 receptors tonically inhibits $\mathrm{TH}$ expression and acts in a paracrine manner to regulate adrenal synaptic signaling $(20,21)$. NPY is a good candidate for mediating the hypoglycemia-induced change in $\mathrm{TH}$, because $\mathrm{Y} 1$ receptors are $\mathrm{G}_{\mathrm{i} / \mathrm{o}}$ coupled, and their activation can suppress TH transcription (18). When we quantified NPY immunoreactivity, we found that expression of NPY was significantly elevated after both single and recurrent hypoglycemic episodes (Figure 3, A and B). If the sustained increase in NPY is responsible for inhibiting TH expression, then preventing NPY signaling should remove the inhibition. First, TH immunoreactivity was quantified in NPY-KO mice. In these animals, the levels of TH immunoreactivity were elevated after both single and recurrent hypoglycemic episodes (Figure 3C). Group data confirmed that hypoglycemia led to an increase in mean TH immunoreactivity and a rightward shift in the cumulative intensity distribution (Figure 3D). Second, animals were injected with BIBP3226, a Y1 antagonist that does not cross the blood-brain barrier, before each saline or insulin injection. In these animals, both single and recurrent hypoglycemic episodes led to increased TH immunoreactivity and a significant rightward shift in the cumulative intensity distribution (Figure 3, E and F), mimicking the effect seen in NPY-KO mice. Thus, the ability of recurrent hypoglycemia to increase TH occurs only in the absence of NPY signaling, indicating that this neuropeptide actively inhibits the adrenal component of the CRR.

Measurement of urine epinephrine levels from NPY-KO mice exposed to the hypoglycemic protocols (Figure 3G) revealed 2 features of interest. First, basal levels were substantially higher than in C57/BL6 mice (Figure 1B). Although a direct comparison is not possible because of different strain backgrounds, the elevated level probably reflects the increased basal expression of $\mathrm{TH}$ in NPY-KO mice (20). Second, recurrent hypoglycemia did not lead to suppressed epinephrine release, even though hypoglycemia was present after each insulin injection (Figure $3 \mathrm{H}$ ). Normalizing the data by comparing the hypoglycemia-induced release relative to saline-injected controls showed that removing NPY prevented the depression of epinephrine release that is the characteristic feature of HAAF (Figure 3I).
Inhibition of chromaffin cell function does not reflect widespread peripheral impairment of the CRR. Since recurrent hypoglycemia evidently alters adrenal function, do other peripheral changes also contribute to the impaired CRR? Chromaffin cells are unusual neural cells that proliferate in adulthood (22). A decrease in cell numbers could theoretically lead to reduced epinephrine release. Apoptotic cell death in the arcuate nucleus is increased by hypoglycemia and may contribute to a suppressed CRR (23). However, measuring proliferation by staining for proliferating cell nuclear antigen (PCNA) revealed that most adrenal PCNA immunoreactivity was in the region of the zona glomerulosa, and there was no change in the number of immunoreactive cells in the medulla following hypoglycemia (Figure 4, A and C). Adrenal sections were also stained for caspase-3, a marker of apoptosis. Immunoreactivity was primarily located in a juxtamedullary region, with occasional cells in the medulla. We detected no difference in the number of caspase-3-immunoreactive cells in the medulla after hypoglycemia (Figure 4, B and D). Thus, the blunted $\mathrm{TH}$ expression and epinephrine release after recurrent hypoglycemia was not due to altered chromaffin cell proliferation or apoptosis.

We next considered whether changes occurred downstream from the adrenal. Hepatic glucose production plays an important role in recovery from IIH (24) and can be measured using the pyruvate tolerance test. However, we detected no relative difference in the kinetics of glucose production in WT mice after pyruvate injection (Figure 4E) or in the area under the glucose response curves (Figure 4G). Likewise, in NPY-KO mice, we observed no difference in hepatic glucose production between the treatments (Figure 4, F and $\mathrm{H}$ ). We detected a small increase in hepatic glycogen levels in WT mice after recurrent hypoglycemia (Figure 4 , I and J), but this cannot explain the impaired CRR, because hepatic glycogen loading increases, rather than decreases, glucose output and circulating epinephrine levels (25). Liver cryosections were also stained using the periodic acid-Schiff reaction, which labels polysaccharides including glycogen. When staining (Figure $4 \mathrm{~K}$ ) was quantified, we found no difference between euglycemic mice and those exposed to hypoglycemia ( $P>0.85, n=3-4$ mice per treatment). Thus, the peripheral defect in the CRR is selective, since it manifests as an observable change in adrenal but not hepatic function.

Here, we have tested the hypothesis that impairment of the CRR after recurrent activation involves peripheral sympatho-adrenal plasticity $(20,21)$. We found that repeated hypoglycemic episodes led to a reduction in optogenetically evoked catecholamine release. Because the effect was present in isolated chromaffin cells, this unambiguously localized a functional defect in the CRR to the adrenal gland itself. Single, but not recurrent, episodes of hypoglycemia increased the expression of $\mathrm{TH}$, and thus the effect may be due to an impairment in refilling the releasable stores of catecholamines that becomes more marked each time the CRR is activated. Loss of NPY (an adrenal cotransmitter) or preventing Y1 signaling rescued the naive phenotype (perhaps by removing a negative regulator of TH transcription; ref. 18). Because other hormonal com- 
A
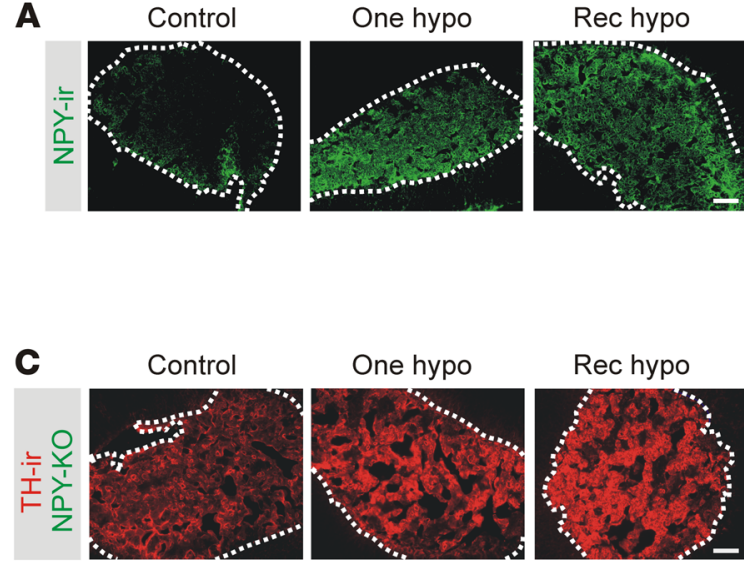

E
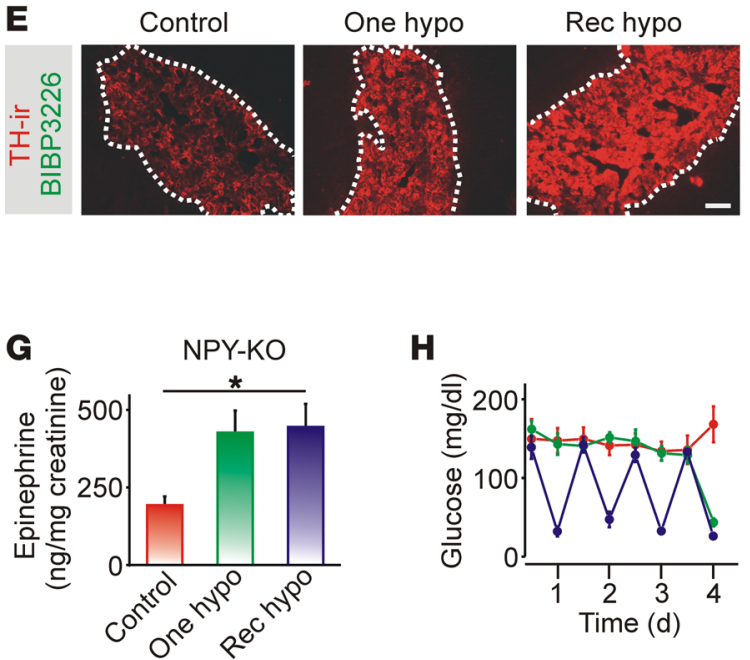

B
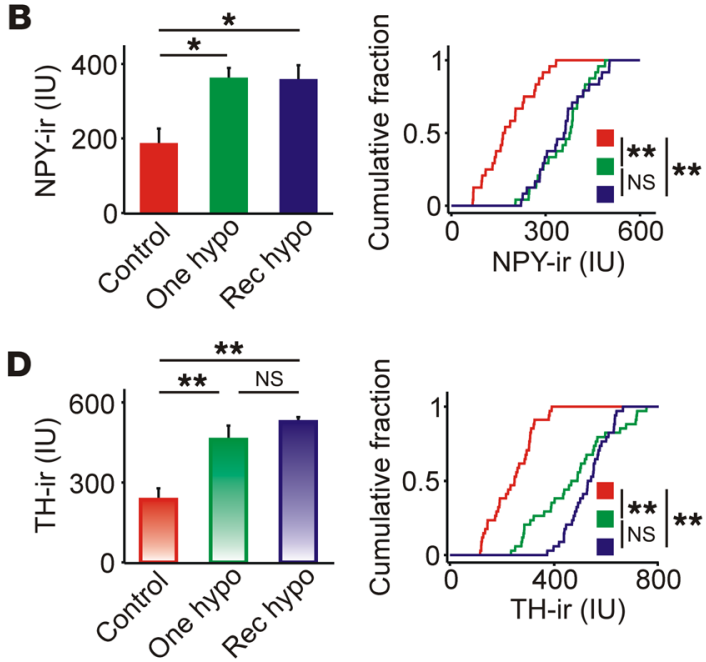

$\mathbf{F}$
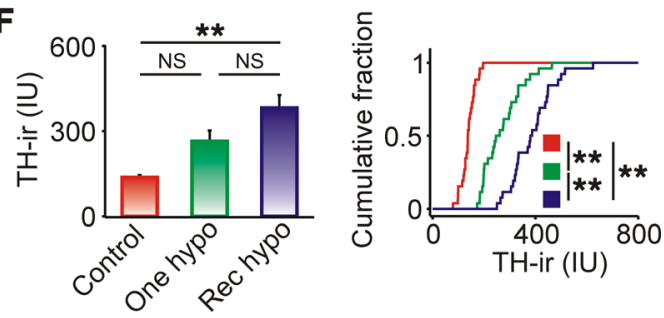

I

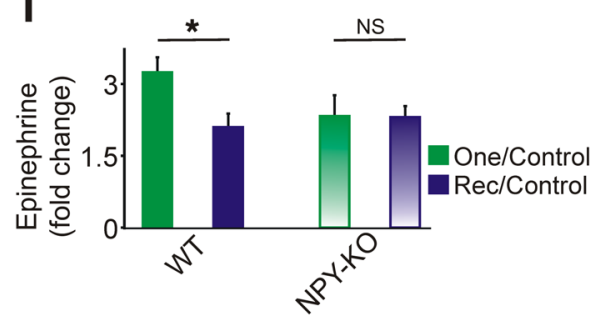

Figure 3. Neuropeptide Y release mediates adrenal impairment of the CRR. (A) NPY immunoreactivity was restricted to the adrenal medulla. (B) NPY immunoreactivity after single and repeated episodes of IIH $(n=3)$ and cumulative intensity distribution of NPY immunoreactivity in all cryosections ( $n=8$ per experiment). (C) Adrenal TH immunoreactivity in NPY-KO mice. (D) TH immunoreactivity was increased after single and recurrent episodes of hypoglycemic $(n=4)$. Right panel shows the cumulative intensity distribution of TH immunoreactivity in all cryosections ( $n=8-10$ per experiment). (E) Adrenal TH immunoreactivity in WT mice injected with BIBP3226. (F) TH immunoreactivity was increased after single and recurrent episodes of hypoglycemia ( $n=3$ ). Right panel shows the cumulative intensity distribution of TH immunoreactivity in all cryosections ( $n=8-10$ per experiment). (G) Urine epinephrine levels were increased after single or recurrent episodes of IIH in NPY-KO mice $(n=4)$. (H) Insulin induced a reproducible fall in blood glucose levels $(n=4)$. (I) Epinephrine release after recurrent IIH was reduced compared with a single episode of hypoglycemia in WT but not NPY-KO mice. ${ }^{*} P<0.05$ and ${ }^{* *} P<0.01$, by 1-way ANOVA and Kolmogorov-Smirnov test (histograms and cumulative distribution graphs, respectively). Scale bars: $100 \mu \mathrm{m}$.

ponents of the CRR are intact in NPY-KO mice (26), we propose a model in which inhibitory paracrine signaling within the adrenal medulla is responsible for the change in secretory capacity and, consequently, the impairment of the CRR (Figure 4L).

The suppression of catecholamine release following recurrent hypoglycemia [1 - (rec hypo/one hypo)] was larger in vivo than in vitro (36\% vs. $24 \%$ ). Thus, in addition to the NPY-dependent reduction in secretory capacity, other changes probably occur. At the level of the adrenal medulla, this may involve a change in preganglionic $\rightarrow$ chromaffin cell transmission $(15,27)$. A reduction in descending outflow is unlikely to contribute, since adrenal nerve activity is not suppressed after recurrent hypoglycemia $(11,12)$. However, further study is needed to determine the relative importance of these different forms of adrenal plasticity. In a wider context, it is curious that adrenergic, opioid, and serotonergic pathways have been implicated in $\operatorname{HAAF}(4-7,28)$ and that these transmitters are also involved in sympatho-adrenal signaling. It seems likely that a variety of peptide and classical transmitters, including NPY, are involved in an autocrine modulation of adrenal epinephrine release.

The decline in the effectiveness of the CRR is long lasting, and scrupulous glycemic control is required for reversal (29). By analogy to the long-lasting changes in synaptic transmission that underlie LTP and memory formation, we can distinguish between the induction and expression of the effects of recurrent hypoglycemia. The induction phase presumably involves glucose sensors in the CNS or portal mesenteric veins that detect the fall in blood glucose (30, 31). Adrenal chromaffin cells are an efferent component and thus involved in the expression of the altered CRR. The latter appears 
A
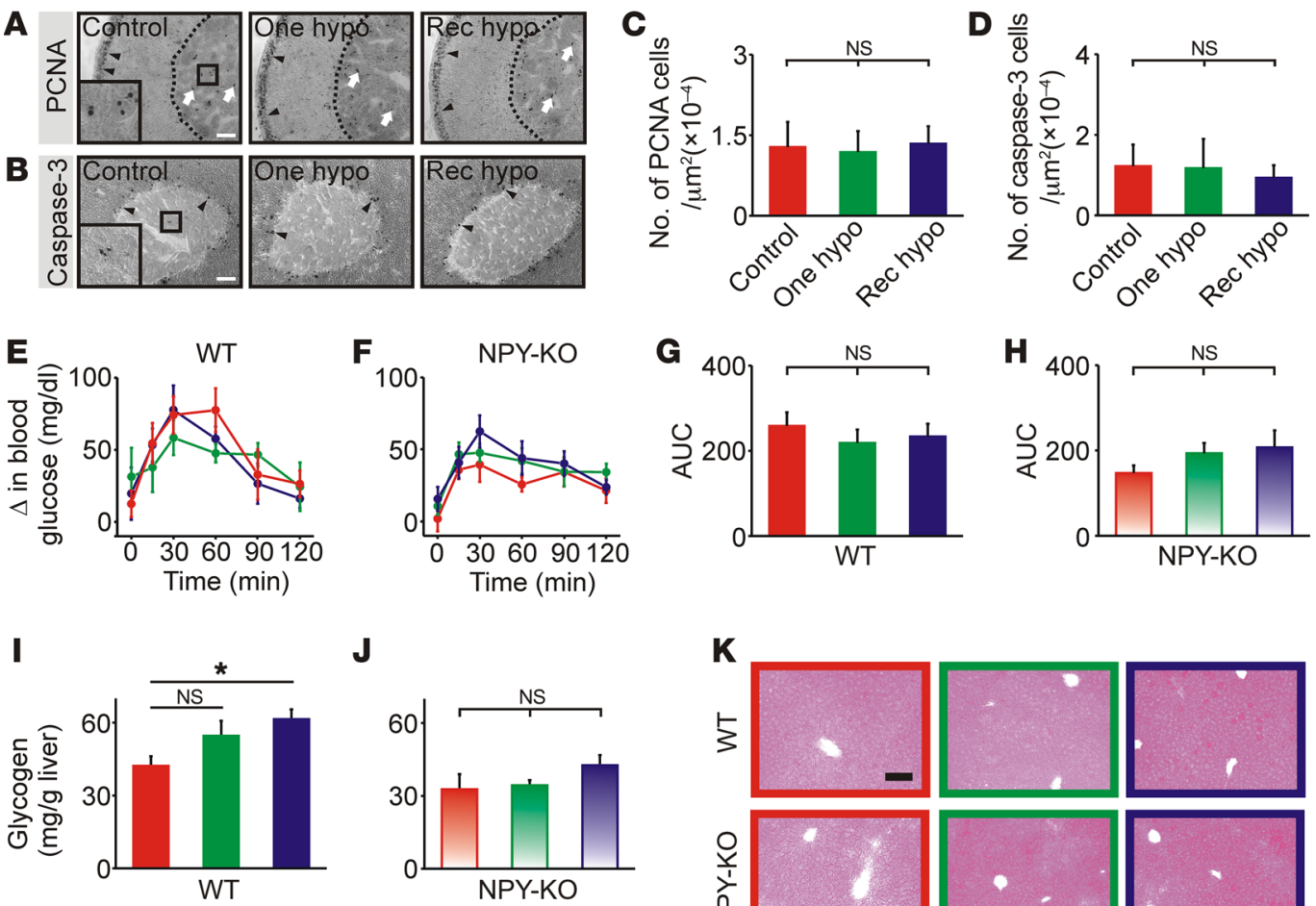

$\mathbf{J}$
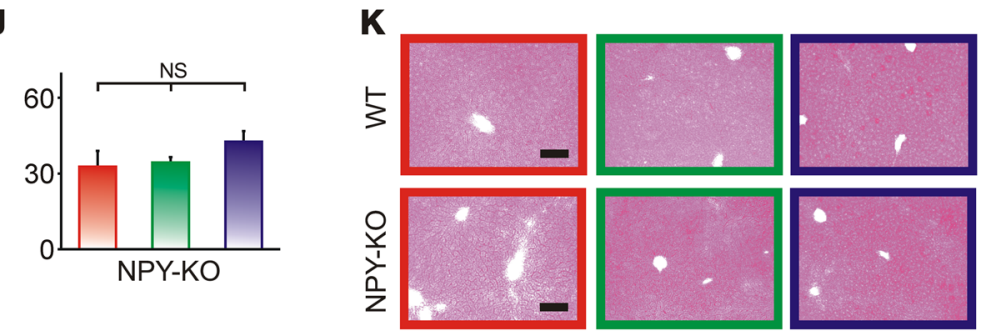

$\mathbf{L}$

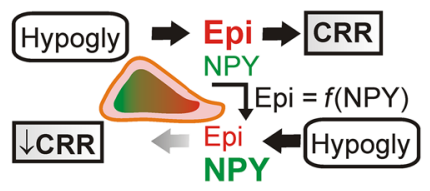

Figure 4. Recurrent hypoglycemia does not lead to widespread peripheral impairment of the CRR. (A) PCNA-immunoreactive cells were mainly found in the region of the zona glomerulosa (black arrowheads), not the medulla (white arrows). (B) Caspase-3-immunoreactive cells (black arrowheads) were present in the cortex. Occasional cells were found in the medulla (boxed area). (C and D) PCNA and caspase-3 immunoreactivity in the adrenal medulla after single or recurrent episodes of IIH ( $n=6$ and 4, respectively). (E and F) Blood glucose levels during pyruvate tolerance tests. (G and $\mathbf{H})$. Quantification of the area under the glucose response curves ( $n=5-6$ per group). (I and J). Hepatic glycogen levels in WT and NPY-KO mice, respectively ( $n=4)$. (K) Periodic acid-Schiff staining in hepatic cryosections. (L) Model of adrenal plasticity during the CRR. Epinephrine (Epi) and NPY are released from chromaffin cells during hypoglycemia (Hypogly) as a part of the CRR. NPY subsequently inhibits the expression of TH, which delays the refilling of the depleted epinephrine-releasable pool; therefore, epinephrine stores are functionally related to the level of NPY release [Epi $=f(N P Y)]$. If hypoglycemia is reexperienced, less epinephrine is available for release, and the magnitude of the CRR is reduced (i.e., HAAF). The inhibition may be further magnified by a hypoglycemia-induced increase in NPY expression. ${ }^{*} P<0.05$, by 1-way ANOVA. Scale bars: $100 \mu \mathrm{m}$.

to involve a change in sympatho-adrenal signaling (11-15; and the present work). Thus, we suggest that the decline in effectiveness of the CRR with repeated use involves multiple loci. Interfering with the peripheral mechanism may be a new approach to avoiding the pathophysiological consequences of recurrent hypoglycemia.

\section{Methods}

Details are provided in the Supplemental Methods.

Statistics. Comparisons between groups containing normally distributed data (Shapiro-Wilk test) were made using ANOVA with Tukey's post hoc test or Student's $t$ test. The Wilcoxon rank-sum test or the Kruskal-Wallis rank-sum test was used for non-normal data, and the Kolmogorov-Smirnov test was used to analyze cumulative distributions. A $P$ value of less than 0.05 was considered significant. Data in the figures represent the mean \pm SEM.

Study approval. All experiments were approved by the IACUC of the Louisiana State University Health Sciences Center (LSUHSC).

\section{Author contributions}

YM performed experiments and contributed to the writing of the manuscript. QW, DJ, and MW performed experiments and reviewed the manuscript. MDW performed experiments and wrote the manuscript. YM, QW, MW, and MDW were involved in study design and data analysis.

\section{Acknowledgments}

This work was supported by NIH grants DK080441 and DK098134 (to MDW). We thank June Liu (LSUHSC) for critically reading the manuscript.

Address correspondence to: Matthew D. Whim, Department of Cell Biology and Anatomy, LSU Health Sciences Center, Medical Education Building (MEB 6142), 1901 Perdido Street, New Orleans, Louisiana 70112, USA. Phone: 504.568.2269; Email: mwhim@1suhsc.edu. 
1. Cryer PE. Glucose counterregulation: prevention and correction of hypoglycemia in humans. Am J Physiol. 1993;264(2 Pt 1):E149-E155.

2. Heller SR, Cryer PE. Reduced neuroendocrine and symptomatic responses to subsequent hypoglycemia after 1 episode of hypoglycemia in nondiabetic humans. Diabetes. 1991;40(2):223-226.

3. Chu CA, Sindelar DK, Neal DW, Allen EJ, Donahue EP, Cherrington AD. Comparison of the direct and indirect effects of epinephrine on hepatic glucose production. JClin Invest. 1997;99(5):1044-1056.

4. Caprio S, et al. Opiate blockade enhances hypoglycemic counterregulation in normal and insulin-dependent diabetic subjects. Am J Physiol. 1991;260 (6 Pt 1):E852-E858.

5. Sanders NM, et al. The selective serotonin reuptake inhibitor sertraline enhances counterregulatory responses to hypoglycemia. Am JPhysiol Endocrinol Metab. 2008;294(5):E853-E860.

6. Leu J, Cui MH, Shamoon H, Gabriely I. Hypoglycemia-associated autonomic failure is prevented by opioid receptor blockade. JClin Endocrinol Metab. 2009;94(9):3372-3380.

7. Ramanathan R, Cryer PE. Adrenergic mediation of hypoglycemia-associated autonomic failure. Diabetes. 2011;60(2):602-606.

8. Song Z, Routh VH. Recurrent hypoglycemia reduces the glucose sensitivity of glucose-inhibited neurons in the ventromedial hypothalamus nucleus. Am J Physiol Regul Integr Comp Physiol. 2006;291(5):R1283-R1287.

9. Chan $\mathrm{O}$, et al. Increased GABAergic tone in the ventromedial hypothalamus contributes to suppression of counterregulatory responses after antecedent hypoglycemia. Diabetes. 2008;57(5):1363-1370.

10. Sanders NM, Ritter S. Repeated 2-deoxy-Dglucose-induced glucoprivation attenuates Fos expression and glucoregulatory responses during subsequent glucoprivation. Diabetes. 2000;49(11):1865-1874.

11. Sivitz WI, Herlein JA, Morgan DA, Fink BD, Phillips BG, Haynes WG. Effect of acute and antecedent hypoglycemia on sympathetic neural activity and catecholamine responsiveness in normal rats. Diabetes. 2001;50(5):1119-1125.

12. Herlein JA, Morgan DA, Phillips BG, Haynes WG, Sivitz WI. Antecedent hypoglycemia, catecholamine depletion, and subsequent sympathetic neural responses. Endocrinology. 2006;147(6):2781-2788.

13. LaGamma EF, Kirtok N, Chan O, Nankova BB. Partial blockade of nicotinic acetylcholine receptors improves the counterregulatory response to hypoglycemia in recurrently hypoglycemic rats. Am J Physiol Endocrinol Metab. 2014;307(7):E580-E588.

14. Kudrick N, et al. Posttranscriptional regulation of adrenal $\mathrm{TH}$ gene expression contributes to the maladaptive responses triggered by insulininduced recurrent hypoglycemia. Physiol Rep. 2015;3(2):e12307.

15. Orban BO, Routh VH, Levin BE, Berlin JR. Direct effects of recurrent hypoglycaemia on adrenal catecholamine release. Diab Vasc Dis Res. 2015;12(1):2-12

16. Galassetti P, Davis SN. Effects of insulin per se on neuroendocrine and metabolic counter-regulatory responses to hypoglycaemia. Clin Sci. 2000;99(5):351-362.

17. Vietor I, Rusnak M, Viskupic E, Blazicek P, Sabban EL, Kvetnansky R. Glucoprivation by insulin leads to trans-synaptic increase in rat adrenal tyrosine hydroxylase mRNA levels. Eur J Pharmacol. 1996;313(1-2):119-127.

18. Cavadas $\mathrm{C}$, et al. Deletion of the neuropeptide $\mathrm{Y}$ (NPY) Y1 receptor gene reveals a regulatory role of NPY on catecholamine synthesis and secretion. Proc Natl Acad Sci U S A. 2006;103(27):10497-10502.

19. Whim MD. Near simultaneous release of classical and peptide cotransmitters from chromaffin cells. J Neurosci. 2006;26(24):6637-6642.

20. Wang $Q$, Wang M, Whim MD. Neuropeptide y gates a stress-induced, long-lasting plasticity in the sympathetic nervous system. J Neurosci. 2013;33(31):12705-12717.

21. Wang $M$, Wang $Q$, Whim MD. Fasting induces a form of autonomic synaptic plasticity that prevents hypoglycemia. Proc Natl Acad Sci U S A.
2016;113(21):E3029-E3038.

22. Verhofstad AA. Kinetics of adrenal medullary cells. J Anat. 1993;183(Pt 2):315-326.

23. Tkacs NC, Dunn-Meynell AA, Levin BE. Presumed apoptosis and reduced arcuate nucleus neuropeptide $\mathrm{Y}$ and pro-opiomelanocortin mRNA in non-coma hypoglycemia. Diabetes. 2000;49(5):820-826.

24. Frizzell RT, et al. Role of gluconeogenesis in sustaining glucose production during hypoglycemia caused by continuous insulin infusion in conscious dogs. Diabetes. 1988;37(6):749-759.

25. Winnick JJ, et al. Hepatic glycogen can regulate hypoglycemic counterregulation via a liver-brain axis. J Clin Invest. 2016;126(6):2236-2248.

26. Sindelar DK, et al. Neuropeptide $\mathrm{Y}$ is required for hyperphagic feeding in response to neuroglucopenia. Endocrinology. 2004;145(7):3363-3368.

27. Hamelink C, et al. Pituitary adenylate cyclaseactivating polypeptide is a sympathoadrenal neurotransmitter involved in catecholamine regulation and glucohomeostasis. Proc Natl Acad Sci U S A. 2002;99(1):461-466.

28. Kim JL, La Gamma EF, Estabrook T, Kudrick N, Nankova BB. Whole genome expression profiling associates activation of unfolded protein response with impaired production and release of epinephrine after recurrent hypoglycemia. PLoS One. 2017;12(2):e0172789.

29. Fanelli CG, et al. Meticulous prevention of hypoglycemia normalizes the glycemic thresholds and magnitude of most of neuroendocrine responses to, symptoms of, and cognitive function during hypoglycemia in intensively treated patients with short-term IDDM. Diabetes. 1993;42(11):1683-1689.

30. Fujita S, Donovan CM. Celiac-superior mesenteric ganglionectomy, but not vagotomy, suppresses the sympathoadrenal response to insulin-induced hypoglycemia. Diabetes. 2005;54(11):3258-3264.

31. Garfield AS, et al. A parabrachial-hypothalamic cholecystokinin neurocircuit controls counterregulatory responses to hypoglycemia. Cell Metab. 2014;20(6):1030-1037. 